\title{
Measurements and analysis of acoustic properties of drainage asphalt
}

\author{
Hisashi Hatanaka and Kohei Yamamoto \\ Kobayasi Institute of Physical Research, \\ 3-20-41, Higashi-Motomachi, Kokubunji, 185-0022 Japan
}

(Received 6 June 1998)

\begin{abstract}
Acoustic properties of drainage asphalt are investigated to simulate the noise propagation of road traffic noise. Normalized acoustic impedance, porosity and flow resistivity are measured by an ordinary method with a small sample of maximum size of aggregate $13 \mathrm{~mm}$ and thickness of $45 \mathrm{~mm}$. By referring to Allard's impedance model for rigid frame porous material and curve fitting technique, tortuosity of the sample is deduced. It is shown that this model gives a good fitting to normalized acoustic impedance measured and applicable to simulate the noise propagation on the drainage asphalt of road surface by a boundary element method. Next, effective flow resistivity is deduced approximately by a short range sound propagation over a drainage asphalt surface of a highway in operation. It is shown an empirical one-parameter impedance model is applicable to the prediction of noise propagation on the drainage asphalt surface by using an effective flow resistivity. Normal and random incident absorption coefficients are measured and presented for data supplements.
\end{abstract}

Keywords : Drainage asphalt, Normalized acoustic impedance, Road traffic noise, Absorption coefficient, Ground impedance

PACS number: 43. 28. En

\section{INTRODUCTION}

Drainage asphalt has been coming into use to road pavements since the 1980's as a hopeful counter measure against road traffic noise in this country. Many research works ${ }^{1-5)}$ have devoted to examine the noise reducing mechanism and to develop new designs for pavements with high benefits. The noise reducing ability has been explained by the two ways, i.e., (a) tire/road noise is reduced at the source position due to the reduction of air pumping noise, and (b) both power unit noise and tire/road noise are decreased during propagation over the pavement. The latter tells us that the surface of the drainage asphalt works as a finite impedance boundary and it absorbs vehicle noise emitted from power units (engine, air intake, exhaust, transmission) and tire/road surfaces.

Therefore the acoustic properties of drainage asphalt, such as characteristic acoustic impedance and absorption coefficient, are important factors for the noise control design of road structures such as noise barriers. For example one should consider the normalized acoustic impedance of a road surface boundary when the noise simulation by Boundary Element Method (BEM) is applied to calculate the insertion loss of a noise barrier erected at the road side. In another case, one may need to know the random incident absorption coefficient for the control of reverberation phenomena inside road tunnel for the security purposes on emergency. Normal and oblique incident absorption coefficients may also be needed in some noise control situation.

In this paper, it is the objective to find the best fit impedance model and the values of parameters for drainage asphalt which has been widely applied to road pavements. For data supplements, using samples of drainage asphalt, absorption coefficient by a 
reverberation room method and normal incident absorption coefficient by a tube method are measured and the results are presented.

\section{IMPEDANCE MODELS FOR ROAD SURFACES}

\subsection{One-parameter Empirical Model}

In prediction of road traffic noise propagation, surfaces of dense asphalt and concrete which are impervious to water are treated as a rigid reflective boundary. This treatment may be reasonable when the prediction model is a simplified and conventional one. ${ }^{6)}$ However, if the prediction is essentially based upon a wave theory, one should specify the normalized acoustic impedance on boundary surfaces. For the ground impedance, Chessell ${ }^{7)}$ suggested the use of the empirical formula for fibrous materials which Delany and Bazley ${ }^{8)}$ originally reported as a result of their measurement. This impedance model is very convenient because it is specified by only a single parameter, i.e., flow resistivity. Note that this parameter is called effective flow resistivity when it is deduced from a short range measurement of sound propagation over a medium boundary. In this application, Isei, et $a l^{9,10)}$ determined values of the effective flow resistivity for representative ground surfaces and built up a table for application. They obtained the value of $20,000 \mathrm{kPa} \cdot \mathrm{s} / \mathrm{m}^{2}$ for dense asphalt and concrete surfaces. This empirical model has been widely applied to calculate surface impedance of media with rigid frame such as ground and pavement for road traffic noise prediction by the well-established theoretical models ${ }^{6)}$ and BEM. ${ }^{11)}$

$\mathrm{Miki}^{12)}$ revised the Delany and Bazley's formulae according to the positive-real property of impedance function and obtained corrected formulae using the Delany and Bazley's experimental data. The corrected formula for normalized characteristic impedance $Z_{\mathrm{s}}$ (time factor: $i \omega t$ ) is as follows,

$$
Z_{\mathrm{s}}=\left\{1+0.07(f / \sigma)^{-0.632}-i\left[0.107(f / \sigma)^{-0.632}\right]\right\},
$$

where $f$ and $\sigma$ are frequency and flow resistivity, respectively. For a rigidly backed porous layer of depth $d$, the normalized impedance $Z(d)$ is given by,

$$
Z(d)=Z_{\mathrm{s}} \operatorname{coth}(i k d),
$$

where $k$ is propagation constant and given by,

$$
k=\left(\omega / c_{0}\right)\left\{0.160(f / \sigma)^{-0.618}+i\left[0.109(f / \sigma)^{-0.618}\right]\right\} .
$$

The term $\omega$ is angular frequency and $c_{0}$ is speed of sound in air.

\subsection{Multi-parameter Theoretical Model}

The surface of drainage asphalt is pervious to water and the structure of the pavement is regarded as porous material with rigid frame. For the ground with porous media such as soil, sand, and loam, Attenborough ${ }^{13)}$ derived four-parameter rigid porous model to provide the relation between impedance and frequency. The parameters are flow resistivity, porosity, grain shape factor and pore shape factor ratio. The former two are independently measurable by non acoustic techniques. The latter two are not directly determined by physical measurements. Note that the appropriate values of grain shape factor and pore shape factor ratio may be deduced by trial-and-error fitting techniques.

On the other hand, Allard ${ }^{14)}$ proposed another theoretical model which employed basically three parameters, i.e., flow resistivity, porosity and tortuosity. The tortuosity has the particular value defined by $(1 / \cos \theta)^{2}$ for a medium containing parallel cylindrical pores inclined at angle $\theta$ to the surface normal as shown in Fig. 1. This is also unmeasurable, but it may be an adjustable parameter and can be deduced by a best-fitting technique. In his model, normalized characteristic impedance is expressed by,

$$
Z_{\mathrm{s}}=\frac{(K \rho)^{1 / 2}}{\rho_{0} C_{0}}, \quad k=\omega(\rho / K)^{1 / 2},
$$

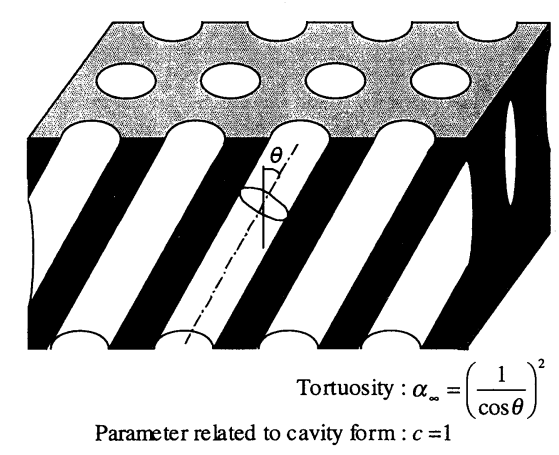

Fig. 1 Model of a porous material with cylindrical pore structure of rigid frame (Allard's model). Parameters are flow resistivity $(\sigma)$, porosity $(\phi)$ and tortuosity $\left(\alpha_{\infty}\right)$. 
where, $\rho$ is effective density, $K$ is bulk modulus, and $\rho_{0}$ is pneumatic density. The effective density $\rho$ and the bulk modulus $K$ are calculated by,

$$
\begin{gathered}
\rho=\alpha_{\infty}\left(\rho_{0}+\frac{\sigma \phi}{i \omega \alpha_{\infty}} G(s)\right), \\
K=\rho_{0} c_{0}^{2} /\left[\gamma-(\gamma-1)\left[1+\frac{8}{i s^{2} B^{2}} G(B \cdot s)\right]^{-1}\right],
\end{gathered}
$$

where $\alpha_{\infty}$ and $\phi$ are tortuosity and porosity, respectively, and with $\gamma$ (specific heat at constant pressure/ specific heat at a constant volume) $=1.4$ and $B^{2}$ (Prandtle number $)=0.72$. The parameter $s$ is given by,

$$
s=c\left(\frac{8 \omega \alpha_{\infty} \rho_{0}}{\phi \sigma}\right)^{1 / 2},
$$

and the function $G(s)$ is expressed as the next expression,

$G(s)=-\frac{s \sqrt{-i}}{4} J_{1,0}(s \sqrt{-i}) /\left[1-\frac{2}{s \sqrt{-i}} J_{1,0}(s \sqrt{-i})\right]$,

where $J_{1,0}(x)=J_{1}(x) / J_{0}(x)$ (Bessel function) and $c$ in Eq. (7) is a parameter related to the shape of a cavity. In the case the cavity form is cylinder, the parameter $c=1 .^{14)}$ If the porous medium is a layer with a depth of $d$ and backed with rigid surface, the normalized impedance $Z(d)$ is expressed by,

$$
Z(d)=\frac{Z_{\mathrm{s}}}{\phi} \operatorname{coth}(i k d) .
$$

It is noted that this formula is the same as that of Eq. (2) except the term of $\phi$. This correction is made according to the continuity of air flow between open air and inside pores of the medium.

The structure of the drainage asphalt consists of rigid particles with various size and form, whereas the multi-parameter theoretical models are derived from an assumption of simple form pores. Considering the simplicity of a theoretical model with only one unmeasurable parameter, Allard's model is employed for the study of acoustic properties of drainage asphalt in this paper. Due to the fact that one parameter empirical model which is derived from acoustical properties of fibrous materials is widely applied to the prediction of road traffic noise, the corrected model of Delany and Bazley is also employed in this study.

\section{SPECIFICATION OF DRAINAGE ASPHALT}

The drainage asphalt of interest in this study was supplied from Japan Highway Public Corporation. It has been widely used to construct highways. The pervious structure is made from maximum particle size $13 \mathrm{~mm}$ of aggregate and designed to have good drainage properties. The test sample supplied was a small specimen with a diameter of $100 \mathrm{~mm}$ and with $45 \mathrm{~mm}$ thickness.

The flow resistivity was measured by the device of air flow tube, ${ }^{15)}$ and the porosity was determined from the weight of the sample and density of the aggregate. Three samples were tested. Since one of them showed a quite different value of flow resistivity, it was excluded from our study. The resulting mean values are $4.7 \mathrm{kPa} \cdot \mathrm{s} / \mathrm{m}^{2}$ for flow resistivity and 0.27 for porosity.

\section{SENSITIVITY ANALYSIS AND IMPEDANCE FITTINGS}

\subsection{Sensitivity Analysis}

Prior to impedance fittings, sensitivity analysis was carried out by using Allard's model. The ranges of the values in the parameter were chosen from close values obtained by measurements, i.e., $\sigma=2,5,8 \mathrm{kPa} \cdot \mathrm{s} / \mathrm{m}^{2}$, and $\phi=0.3,0.5,0.8$. For tortuosity $\left(\alpha_{\infty} \geqq 1\right)$, trial values were selected as $\alpha_{\infty}=$ 3, 8, 13. As for the parameter $c$ in Eq. (7), a simple value of 1 was specified on the assumption of cylindrical form pores. From Eqs. (4) to (9) and with the depth $d=45 \mathrm{~mm}$, real and imaginary parts of normalized impedance were calculated and shown in Figs. 2 to 4.

Figure 2 shows the sensitivity of the normalized impedance to the assumed values of $\sigma$. In the range

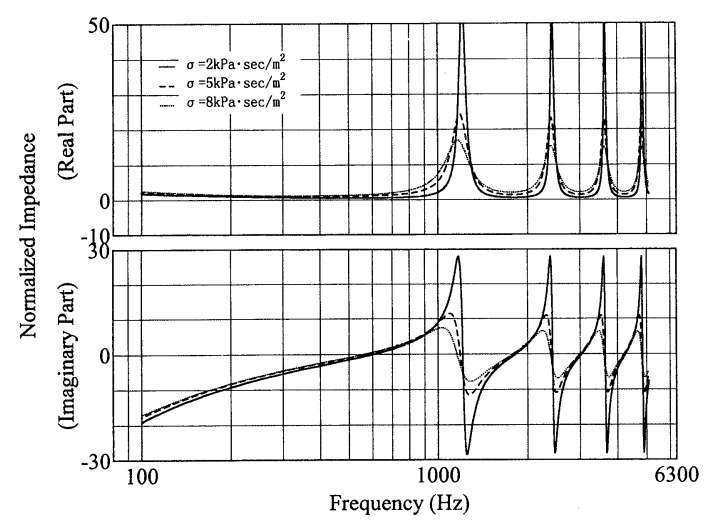

Fig. 2 Effect of flow resistivity ( $\sigma$ ) on normalized acoustic impedance of drainage asphalt with the parameters ; $\phi=0.5, \alpha_{\infty}=8.0$. 


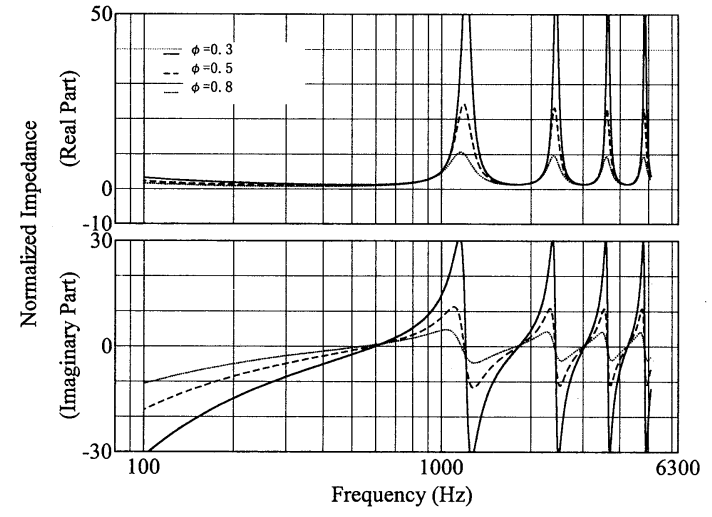

Fig. 3 Effect of porosity $(\phi)$ on normalized acoustic impedance of drainage asphalt with the parameters ; $\sigma=5 \mathrm{kPa} \cdot \mathrm{s} / \mathrm{m}^{2}, \alpha_{\infty}=8.0$.

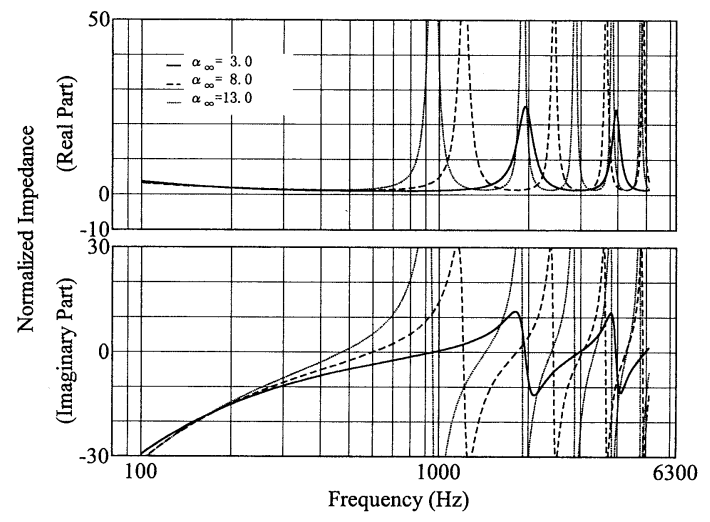

Fig. 4 Effect of tortuosity $\left(\alpha_{\infty}\right)$ on normalized acoustic impedance of drainage asphalt with the parameters ; $\sigma=5 \mathrm{kPa} \cdot \mathrm{s} / \mathrm{m}^{2}, \phi_{\infty}=0.5$.

of our study, great sensitivity is not obtained below $1,000 \mathrm{~Hz}$ both in real and imaginary part. However, it is sensible at the frequencies above $1,000 \mathrm{~Hz}$ with peaks and dips. Figure 3 shows the same comparison with regard to the values of $\phi$. It is shown that the sensitivity in imaginary part is greater than that in real part. By contrast to Figs. 2 and 3, great sensitivity is exhibited to variation in tortuosity as shown in Fig. 4. Peaks and dips are shifted to lower frequencies as the values of $\alpha_{\infty}$ increases. Of the three parameters in the reference range, normalized surface impedance is most sensible to variations in tortuosity.

\subsection{Curve Fittings to Impedance}

It would be useful to give an appropriate value for

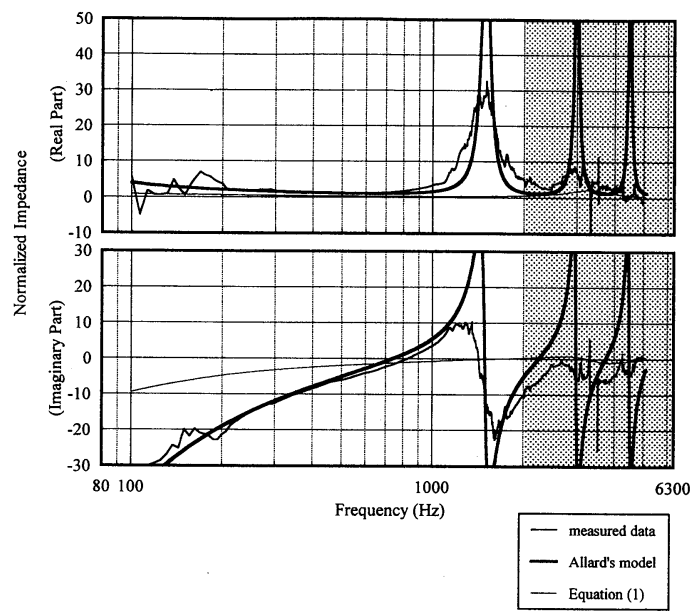

Fig. 5 Comparison of Allard's model prediction for $\sigma=4.7 \mathrm{kPa} \cdot \mathrm{s} / \mathrm{m}^{2}, \phi=0.27$ and $\alpha_{\infty}=$ 6.5 with the measured data. Impedance curves by Eqs. (1) to (3) for $\sigma=4.7 \mathrm{kPa} \cdot \mathrm{s} / \mathrm{m}^{2}$ and $d=45 \mathrm{~mm}$ are also shown for comparison.

$\alpha_{\infty}$ that could be obtained by the calculation of best fit value to the measured impedance data. The procedure is to adjust the value to obtain the best-fit curve with the measured impedance data. The normalized impedance of the samples was measured by a two microphone technique ${ }^{16)}$ with an aid of acoustic tube. Impedance curves were calculated from Eqs. (4) to (9) with $\sigma=4.7 \mathrm{kPa} \cdot \mathrm{s} / \mathrm{m}^{2}, \phi=0.27$ and $d=45 \mathrm{~mm}$. The parameter $\alpha_{\infty}$ was adjustable and was varied to obtain the best-fit impedance curve with the measured data. The estimated normalized impedance is shown in Fig. 5 in comparison with the experimental data. The dark zone indicates the frequency range beyond the reliable region related to the frequency limit of the acoustic tube. It is shown that good fittings are obtained. The tortuosity deduced by the fitting is a value of 6.5 .

In Fig. 5, the impedance curves which are calculated from Eqs. (1) to (3) with $\sigma=4.7 \mathrm{kPa} \cdot \mathrm{s} / \mathrm{m}^{2}$ and $d=45 \mathrm{~mm}$ are also shown. It is noted that there is a remarkable difference between the measured and the calculated impedance. Bolen and Bass ${ }^{17)}$ reported that the use of a flow resistivity of approximately half the mean measured values gave the same values of effective flow resistivity that was obtained from the best-fit of the empirical single parameter predictions to the impedance values deduced from indirect measurement. They treated various 
grounds as a homogeneous and locally reacting porous medium, and the flow resistivity was several hundred $\mathrm{kPa} \cdot \mathrm{s} / \mathrm{m}^{2}$. However, the measured flow resistivity of the present medium, i.e., drainage asphalt, is approximately $1 / 100$ of the values measured by Bolen and Bass. It is the next subject of interest to investigate the effective flow resistivity of the drainage asphalt.

\section{EFFECTIVE FLOW RESISTIVITY OF DRAINAGE ASPHALT AT A HIGHWAY}

As is described in the previous section, the measured flow resistivity of drainage asphalt is very low in comparison with ordinary ground. The value is almost similar to that of light glass wool $(10 \mathrm{kPa} \cdot \mathrm{s})$ $\mathrm{m}^{2}$ ). Considering a practical simulation of long distance sound propagation over drainage asphalt, effective flow resistivity $\sigma_{\mathrm{e}}$ is deduced at a real highway by the same technique as is made for grounds. The effective flow resistivity is determined by curve fittings to the excess attenuation which is obtained from short range measurement of sound propagation over the surface. Since it was unlikely that there would be a precise information of the depth $d$ of drainage asphalt, the effective flow resistivity was deduced by using only Eq. (1), i.e., one-parameter empirical model. Excess attenuation was calculated by a theoretical model for sound propagation over finite impedance boundary. ${ }^{18)}$

The test site was selected at a highway in operation, where drainage asphalt was laid. The pavement was the same grade as was described earlier. The depth of the drainage asphalt was said to be 45 $\mathrm{mm}$, but it was unable to check by a measurement. The age of the surface was three months after the time when the drainage asphalt was first laid. The short range measurement of sound propagation was carried out with a source height $H_{\mathrm{s}}=0.51 \mathrm{~m}$, microphone height $H_{\mathrm{r}}=0.51 \mathrm{~m}$ and the horizontal separation between the source and the microphone $R=$ $10.0 \mathrm{~m}$. The excess attenuation was determined by the attenuation in excess of that due to spherical spreading in free field.

In Fig. 6, a predicted excess attenuation curve and the measured data are shown. There are discrepancies in the measured data at low frequencies, which might be caused by an influence of high level background noise. Good fittings between the predicted curve and the measured data are obtained

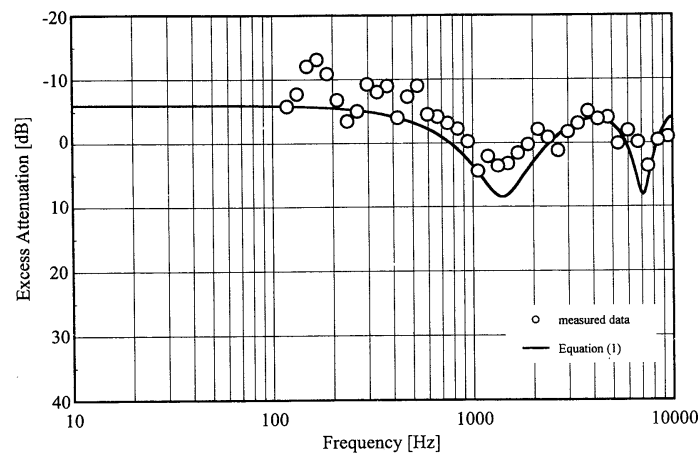

Fig. 6 Comparison of the empirical oneparameter model prediction of excess attenuation for $\sigma=1,000 \mathrm{kPa} \cdot \mathrm{s} / \mathrm{m}^{2}$ with the data measured for drainage asphalt surface at a real highway.

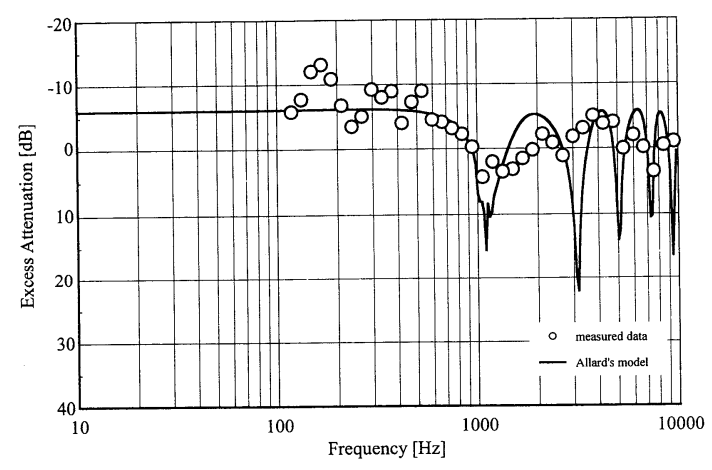

Fig. 7 Comparison of Allard's model prediction of excess attenuation for $\sigma=4.7 \mathrm{kPa} \cdot \mathrm{s} /$ $\mathrm{m}^{2}, \phi=0.2, \alpha_{\infty}=8.0$ and $d=20 \mathrm{~mm}$ with the data measured for drainage asphalt surface at a real highway.

above $2,500 \mathrm{~Hz}$, but the curve shifts downward parallel to the measured data below $2,000 \mathrm{~Hz}$. The value of effective flow resistivity was deduced from a value of the first maximum on the frequency axis. The approximate value of $1,000 \mathrm{kPa} \cdot \mathrm{s} / \mathrm{m}^{2}$ is obtained as $\sigma_{\mathrm{e}}$. (This deduced value is 200 times greater than that of the directly measured flow resistivity.)

Another trial fittings are carried out by using Allard's model. The comparison of the measured and predicted curve of the excess attenuation for the same geometry is shown in Fig. 7. In this case, trial-and-error fitting was carried out by specifying $\sigma=4.7 \mathrm{kPa} \cdot \mathrm{s} / \mathrm{m}^{2}$. The resulting deduced values for parameters are $\phi=0.2, \sigma_{\infty}=8$ and $d=20 \mathrm{~mm}$. 
If one compares Fig. 6 with Fig. 7, the fitting in detail, ex. dip frequencies, is rather better in Fig. 7 than in Fig. 6. However, as a rough approximation, one may treat drainage asphalt surface as one of the ground surfaces and may apply the simple model of Eq. (1) to the prediction of long distance propagation for practical purposes.

\section{ABSORPTION COEFFICIENTS}

With the aid of Allard's impedance model and the values of parameters obtained earlier, we can easily predict $^{19)}$ various absorption coefficients. In this section, absorption coefficients of normal incident by the standing wave ratio method and the reverberation room method are mentioned for data supplements and they are compared with predicted values.

\subsection{Normal Incident Absorption Coefficient by the Standing Wave Ratio Method}

Normal incident absorption coefficients of the drainage asphalt of small samples were measured by the standing wave ratio method (SWR). The absorption coefficient (open circle) versus frequency in $1 / 3$ octave band center frequency is shown in Fig. 8. A remarkable maximum in absorption coefficient is found at $800 \mathrm{~Hz}$. Two predicted curves are drawn in the same figure. One is calcu-

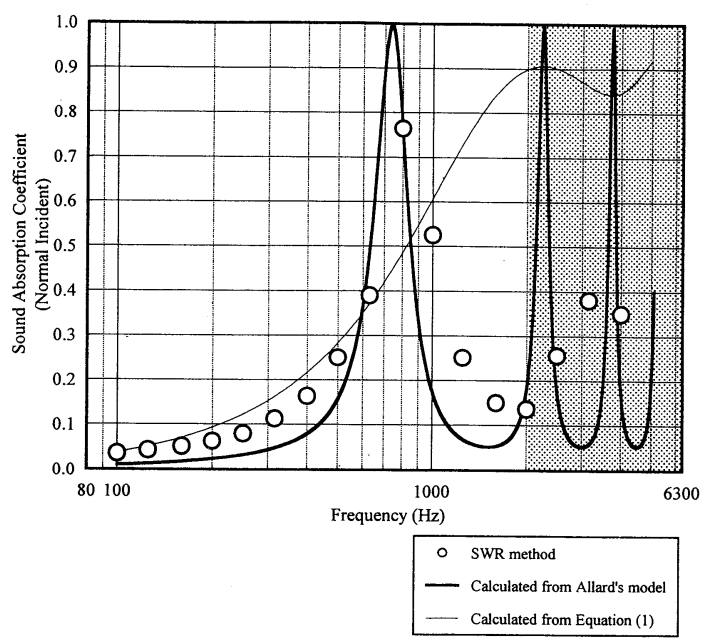

Fig. 8 Comparison of Allard's model prediction of normal incident absorption coefficient for $\sigma=4.7 \mathrm{kPa} \cdot \mathrm{s} / \mathrm{m}^{2}, \phi=0.27, \alpha_{\infty}=6.5$ and $d=45 \mathrm{~mm}$ with the data measured by the SWR method. One parameter model prediction for $\sigma=4.7 \mathrm{kPa} \cdot \mathrm{s} / \mathrm{m}^{2}$ and $d=45 \mathrm{~mm}$ is also shown.

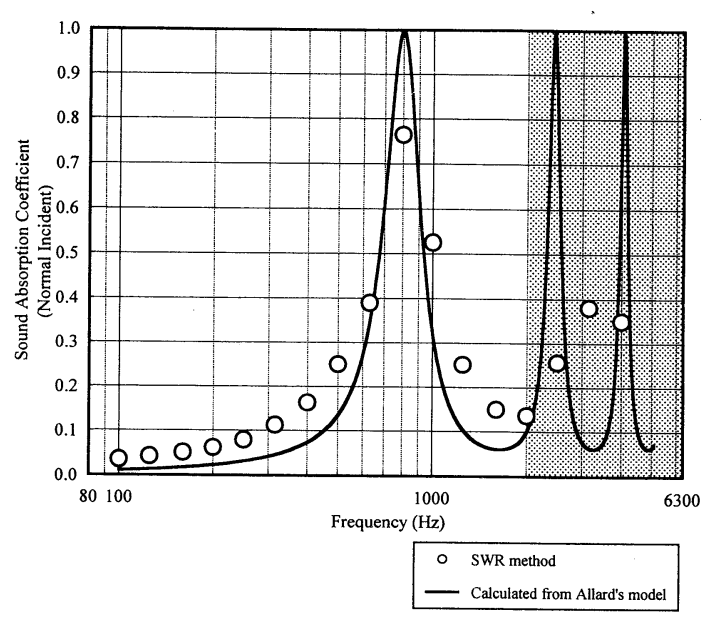

Fig. 9 Comparison of Allard's model prediction of normal incident absorption coefficient for $\sigma=4.7 \mathrm{kPa} \cdot \mathrm{s} / \mathrm{m}^{2}, \phi=0.27, \alpha_{\infty}=5.5$ and $d=45 \mathrm{~mm}$ with the data measured by the SWR method.

lated ${ }^{19)}$ by the use of Allard's impedance model with $\sigma=4.7 \mathrm{kPa} \cdot \mathrm{s} / \mathrm{m}^{2}, \phi=0.27, \alpha_{\infty}=6.5$ and $d=45 \mathrm{~mm}$. The other is by the empirical impedance model of Eqs. (1) to (3) with $\sigma=4.7 \mathrm{kPa} \cdot \mathrm{s} / \mathrm{m}^{2}$ and $d=45$ $\mathrm{mm}$.

It is found that there is a significant difference between the measured and the calculated values above $800 \mathrm{~Hz}$ when predicted by the empirical impedance model with the measured flow resistivity. By contrast, the predicted curve by Allard's model agrees quite well with the measured data. However, it seems the first sharp peak shifts to lower frequency below $800 \mathrm{~Hz}$. Since a local maximum in absorption coefficient is related to the frequency where imaginary part of the normalized impedance crosses the zero line from negative to positive sign where the real part is with a steady value, the shift is due to the predicted error in imaginary part. In fact, one can see the first zero-cross frequency of the imaginary part measured is at around $800 \mathrm{~Hz}$, while predicted curve crosses at $700 \mathrm{~Hz}$ in Fig. 5. One can obtain fairly good agreement for normal incident absorption coefficient by adjusting the tortuosity value from 6.5 to 5.5 , the result of which is shown in Fig. 9.

6.2 Absorption Coefficient by the Reverberation Room Method

Absorption coefficient of the drainage asphalt is 


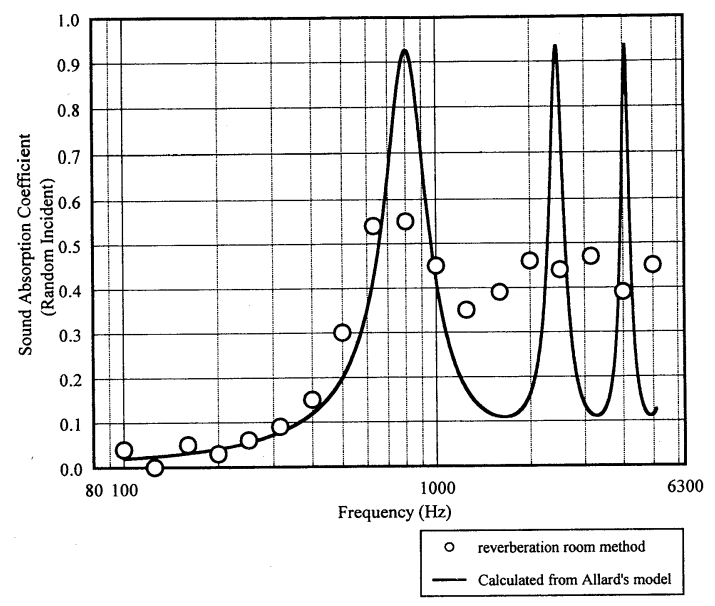

Fig. 10 Comparison of Allard's model prediction of random incident absorption coefficient for $\sigma=4.7 \mathrm{kPa} \cdot \mathrm{s} / \mathrm{m}^{2}, \phi=0.27, \alpha_{\infty}=5.5$ and $d=45 \mathrm{~mm}$ with the data measured by the reverberation room method.

measured by the reverberation room method. The specimen with the same grade as was used to test the normalized impedance and the normal incident absorption coefficient was again supplied from Japan Highway Public Corporation. Small samples in square shape with $500 \mathrm{~mm}$ long were arranged on the floor in rectangle shape with $5.5 \mathrm{~m}$ long and $3.5 \mathrm{~m}$ wide (the total area is $19.25 \mathrm{~m}^{2}$ ). These samples were cut out from a test site pavement, and an average thickness of samples was 45 $\mathrm{mm}$ with a deviation of $\pm 10 \mathrm{~mm}$.

The absorption coefficient determined in the reverberation room with a volume of $513 \mathrm{~m}^{3}$ is shown in Fig. 10. The predicted curve of random incident absorption coefficient ${ }^{19)}$ calculated from Allard's impedance model is also shown in the figure. The parameters specified are $\sigma=4.7 \mathrm{kPa} \cdot \mathrm{s} / \mathrm{m}^{2}, \phi=0.27$, $\alpha_{\infty}=5.5$ and $d=45 \mathrm{~mm}$. The tortuosity selected is the best fit value for the normal incident absorption coefficient.

The absorption coefficient measured (open circle) exhibits local maximum around $700 \mathrm{~Hz}$ to $800 \mathrm{~Hz}$, which corresponds to the frequency predicted. However, the absorption coefficients at these frequencies are much lower than the predicted one. Above $2,000 \mathrm{~Hz}$, there are no remarkable absorption peaks, while sharp peaks at $2,500 \mathrm{~Hz}$ and $4,000 \mathrm{~Hz}$ are observed by prediction.

\section{DISCUSSION}

As was shown in the sensitivity analysis, tortuosity was the most sensible to the normalized impedance and the frequency characteristics of impedance varies critically depending on tortuosity. In the model which is based upon a medium containing parallel cylinder pores, the tortuosity is independently defined as a function of the inclined angle of pores to the surface normal. It is unlikely to make a direct determination of the inclined angles to obtain the tortuosity. Therefore, the curve fitting technique may be useful to deduce this parameter.

If the model specifies the particles of the solid frame rather than that of the pore structure, it may be likely that tortuosity is somewhat related to flow resistivity and porosity. This will be the subject of further investigation.

The flow resistivity measured for small samples of drainage asphalt was very low with a value of 4.7 $\mathrm{kPa} \cdot \mathrm{s} / \mathrm{m}^{2}$. By contrast, the effective flow resistivity of the surface with the same grade drainage asphalt pavement appeared to be a value of $1,000 \mathrm{kPa} \cdot \mathrm{s} / \mathrm{m}^{2}$. Both parameters, i.e., $\sigma$ and $\sigma_{\mathrm{e}}$ have the same role as that in Eq. (1). Note that effective flow resistivity is a parameter for rough deduction of drainage asphalt surface which contains porosity, tortuosity, depth and backed impedance. This parameter is useful only when one treat the road surface as one of the grounds for the prediction of long distance propagation with a well established theory.

The porosity was estimated from the air volume which is obtained by the subtraction of the rigid frame volume to the total volume. The rigid frame volume was calculated by the total weight divided by the density of the aggregate. Therefore, the porosity was not exactly the defined value which should be deduced from the volume of air that is not locked within the frame. For one idea to obtain this volume of air, one may apply an acoustic volume meter ${ }^{20)}$ which was developed for volume of a medium with closed frame.

\section{CONCLUSION}

Acoustic properties of drainage asphalt with a typical structure have been investigated. It has been shown that the Allard's impedance model with values of the parameter gives fairly good fittings to the experimentally obtained normalized impedance. The parameters of flow resistivity and porosity of 
test samples were determined independently by non acoustic technique and obtained values were 4.7 $\mathrm{kPa} \cdot \mathrm{s} / \mathrm{m}^{2}$ and 0.27 , respectively. Tortuosity was deduced by curve fitting technique and a value of 6.5 was obtained.

The effective flow resistivity of the drainage asphalt with the same grade as the test sample was deduced by the short range measurement of sound propagation over a surface of a highway in operation. It has been shown that the deduced effective flow resistivity has an approximate value of 1,000 $\mathrm{kPa} \cdot \mathrm{s} / \mathrm{m}^{2}$ which is 200 times larger than the flow resistivity determined by an air flow tube.

Absorption coefficients are measured by the SWR method and the reverberation room method. It is shown that a typical peak in absorption coefficient appears at $800 \mathrm{~Hz}$, which is consistent with the prediction by Allard's model.

It may be said that the Allard's impedance model can be applicable to the specification of boundary conditions on pavement surface to calculate noise propagation using BEM technique. It may also be said that the empirical impedance model shown in Eq. (1) could be a useful tool to an approximate estimation of long range noise propagation over drainage asphalt. However, the values of unmeasurable parameters such as tortuosity for Allard's model and effective flow resistivity for one parameter empirical model must be supplemented for reliable simulation of road traffic noise.

\section{ACKNOWLEDGEMENTS}

This work was financially supported by Japan Highway Public Corporation. The authors would like to express their gratitude to Mr. Y. Terakado of J.H.P.C. for his efforts of supplying test samples and the arrangement of test site at an expressway. Dr. J. Igarashi helped us with the writing and gave us valuable advice.

\section{REFERENCES}

1) U. Sandberg, "Road traffic noise - The influence of the road surface and its characterization," Appl. Acoust. 21, 97-118 (1987).

2) P. M. Nelson and P. G. Abbott, "Low noise road surfaces," Appl. Acoust. 21, 119-137 (1987).

3) S. Meiarashi, F. Nakashiba, H. Niimi, M. Hasebe, and T. Nakatsuji, "Quantitative comparison between noise reduction factors of drainage asphalt pave- ment," Appl. Acoust. 44, 165-179 (1995).

4) S. Meiarashi, M. Ishida, F. Nakashiba, H. Niimi, M. Hasebe, and T. Nakatsuji, "Improvement in the effect of drainage asphalt road surface on noise reduction," Appl. Acoust. 47, 189-204 (1996).

5) S. Meiarashi, M. Ishida, T. Fujiwara, M. Hasebe, and T. Nakatsuji, "Noise reduction characteristics of porous elastic road surfaces," Appli. Acoust. 47, 239250 (1996).

6) K. Takagi and K. Yamamoto, "Calculation method for road traffic noise propagation proposed by ASJ," Proc. Inter-Noise 94, 289-294 (1994).

7) C. I. Chessell, "Propagation of noise along a finite impedance boundary," J. Acoust. Soc. Am. 62, 825834 (1977).

8) M. E. Delany and E. N. Bazley, “Acoustical properties of fibrous absorbent materials," Appl. Acoust. 3, 105-116 (1970).

9) T. Isei, "Effect of ground surface on noise propagation outdoors," J. Acoust. Soc. Jpn. (J) 38, 270-276 (1982) (in Japanese).

10) T. F. W. Embleton, "Noise propagation outdoors," Lecture Note at National Institute for Resources and Environment (Sept. 1994).

11) D. C. Hothersall, S. N. Chandler-Wilde, and M. N. Hajmirzae, "Efficiency of single noise barriers," J. Sound Vib. 146, 303-322 (1991).

12) Y. Miki, "Acoustical properties of porous materials - Modification of Delany-Bazley models," J. Acoust. Soc. Jpn. (E) 11, 15-24 (1990).

13) K. Attenborough, "Acoustical impedance models for outdoor ground surfaces," J. Sound Vib. 99, 521-544 (1985).

14) J. F. Allard, Propagation of Sound in Porous Media (Elsevier Applied Science, London/New York, 1993), Chap. 5.

15) H. Hatanaka, H. Ogawa, and J. Yoshimura, "Measurements of air flow resistivity of absorbent materials," Proc. Autumn Meet. Acoust. Soc. Jpn. 3-2-7, 807-808 (1991) (in Japanese).

16) J. Y. Chung and D. A. Blaser, "Transfer function method of measuring in-duct acoustics," J. Acoust. Soc. Am. 63, 907-913 (1980).

17) L. N. Bolen and H. E. Bass, "Effects of ground cover on the propagation of sound through the atmosphere," J. Acoust. Soc. Am. 69, 950-954 (1981).

18) S. I. Thomasson, “ A powerful asymptotic solution for sound propagation above an impedance boundary," Acustica 45, 122-125 (1980).

19) H. Kuttruff, Room Acoustics, 2nd Ed. (Applied Science Publishers, London, 1979), Chaps. 2 and 3.

20) K. Taya, J. Kanazawa, H. Komura, and Y. Ishii, "On the development acoustic volume meter," Proc. Autumn Meet. Acoust. Soc. Jpn. 1-6-9, 503-504 (1995) (in Japanese). 